\title{
Charge Competition and the Linear Dynamic Range of Detection in Electrospray Ionization Mass Spectrometry
}

\author{
Keqi Tang, Jason S. Page, and Richard D. Smith \\ Biological Sciences Division and Environmental Molecular Sciences Laboratory, Pacific Northwest National \\ Laboratory, Richland, Washington, USA
}

\begin{abstract}
An experimental investigation and theoretical analysis are reported on charge competition in electrospray ionization (ESI) and its effects on the linear dynamic range of ESI mass spectrometric (MS) measurements. The experiments confirmed the expected increase of MS sensitivities as the ESI flow rate decreases. However, different compounds show somewhat different mass spectral peak intensities even at the lowest flow rates, at the same concentration and electrospray operating conditions. MS response for each compound solution shows good linearity at lower concentrations and levels off at high concentration, consistent with analyte "saturation" in the ESI process. The extent of charge competition leading to saturation in the ESI process is consistent with the relative magnitude of excess charge in the electrospray compared to the total number of analyte molecules in the solution. This ESI capacity model allows one to predict the sample concentration limits for charge competition and the on-set of ionization suppression effects, as well as the linear dynamic range for ESI-MS. The implications for quantitative MS analysis and possibilities for effectively extending the dynamic range of ESI measurements are discussed. (J Am Soc Mass Spectrom 2004, 15, 1416-1423) ( 2004 American Society for Mass Spectrometry
\end{abstract}

$\mathrm{E}$ lectrospray ionization-mass spectrometry (ESIMS) [1] has become a widely used analytical technique in modern biological research due to its high sensitivity and broad applicability $[2,3]$. Because of the extreme complexity of many biological samples (e.g., proteomics analyses), the effectiveness of ESI-MS depends substantially on both its achievable sensitivity and dynamic range. It has been recognized that optimization of ESI processes has a great effect on MS sensitivity [4]. Operating electrosprays in a so called nanoelectrospray [5] mode, with flow rates ranging from 10 to $100 \mathrm{nl} / \mathrm{min}$, has been shown to significantly increase MS sensitivity compared with more conventional electrospray operation $(>1 \mu \mathrm{l} / \mathrm{min})$ [6]. The much smaller charged droplets (nanometer range) generated by nanoelectrosprays $[7,8]$ lead to increased ionization efficiency. It has also been shown that effects upon the analyte ionization efficiency resulting from ionic contaminants (matrix effects) or multiple analytes (ionization suppression) decrease significantly for nano-electrosprays compared to conventional electrosprays $[5,9]$. Although many advantages have been recognized in

Published online August 27, 2004

Address reprint requests to Dr. R. D. Smith, Biological Sciences Division and Environmental and Molecular Sciences Laboratory, Pacific Northwest National Laboratory, MSIN K8-98, P.O. Box 999, Richland, WA 99352, USA. E-mail: rds@pnl.gov principle for nano-electrospray operation, a firm quantitative experimental evaluation in terms of its achievable MS sensitivity is still not available due to factors that include difficulties in reliably operating nano-electrosprays [10]. The most effective nano-electrospray operation requires the interface to be optimized for the significantly smaller sample infusion rate, more facile droplet desolvation, and the lower electrospray current [8].

The linear dynamic range for ESI-MS measurements has been previously investigated resulting in somewhat differing conclusions [11-17]. The upper concentration limit was originally defined by Kebarle and Tang [11, 12] as the one corresponding to the maximum analyte charge limit for a given total electrospray current. Above this concentration limit, the ESI response to the analyte concentration levels off, a phenomenon often referred to as analyte "saturation" [13]. Other experimental studies of the ESI linear dynamic range have reported a fixed upper analyte concentration limit of $\sim 10^{-5}$ Molar, regardless of electrospray operating conditions [14-17].

There are potentially several reasons for the reported differences for the upper concentration limit of the ESI linear dynamic range. The first possibility is related to the reliability of the electrospray current measurement under different operating conditions. As first reported by de la Mora and Loscertales [8], electrosprays of highly conductive aqueous solutions operated in the 


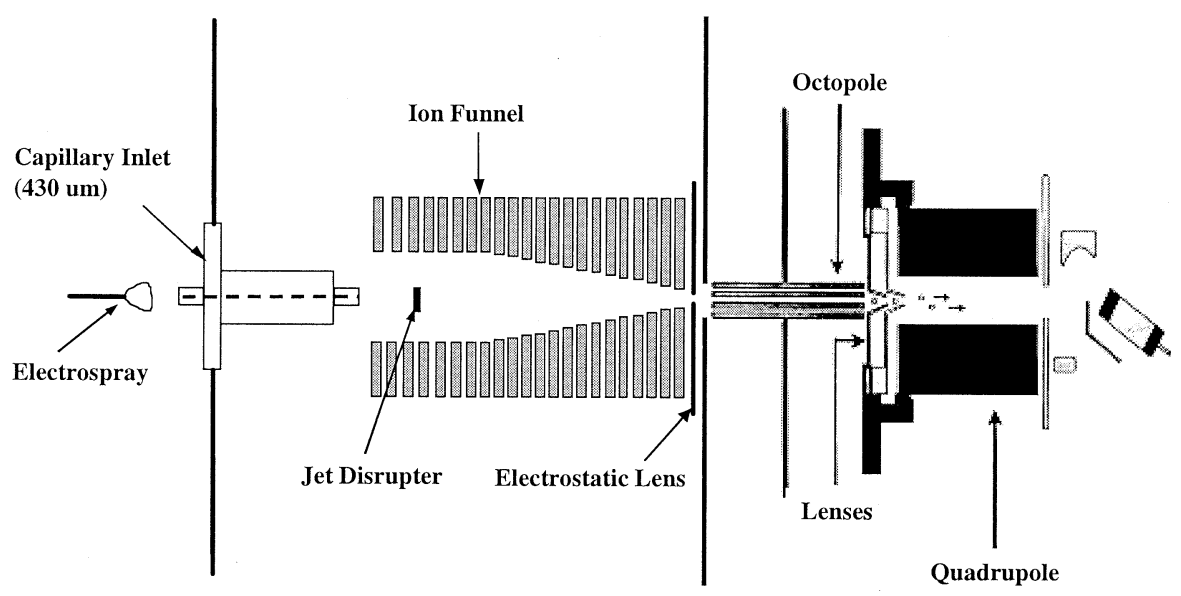

Figure 1. Illustration of the modified Agilent MSD 1100 single quadrupole mass spectrometer used in this work (ion funnel not drawn to scale).

so-called "cone-jet" mode [18], display total electrospray currents independent of both the emitter (tip) diameter and applied voltage, and are dictated only by the liquid flow rate and the relevant liquid properties, such as electric conductivity, surface tension and dielectric constant. Outside the cone-jet mode, the electrospray may display completely different current characteristics, and the charged droplets become significantly polydisperse in size and extent of charging [18-20]. This can potentially lead to significant differences compared to the optimum electrospray performance achievable in the cone jet mode, and errors in the estimation of the 'excess' droplet charge, and causing changes in the maximum concentration for which ESI linear response will be achieved. A second possibility is related to the optimization of the ion sampling and transmission efficiencies through the ESI-MS interface. Due to the high analyte ion density when an electrospray is operated at or above the upper concentration limit for the ESI linear dynamic range, the MS ion sampling and transmission efficiencies may be limited by the space charge effects on the ion optics of the mass spectrometer. Extremely large ion currents could potentially require significant redesign of the ESI interface to avoid ion transmission bottlenecks, so that MS response would more correctly reflect higher ion production rates. A third possibility involves the charge competition effects on the ESI process in the presence of a mixture having high analyte concentrations. Compounds having different proton affinities, surface activities, etc. $[17,21]$ can have different ionization efficiencies, and consequently potentially have different ranges of linear response. Due to the importance of dynamic range for making quantitative measurements in ESI-MS and the different views apparent in the literature, we have revisited this issue.

We report a study of MS response under different electrospray operating conditions and the characteristics of the charge competition in the ESI process when sample mixtures are used for ESI-MS analysis. The linear dynamic range of the ESI process has been investigated using both single analyte and a mixture of analytes with different ionization efficiencies. The experimental measurements of ESI linear dynamic range and theoretical predictions based on the ESI charge capacity are compared. The implications of charge competition for quantitative MS analysis and the possibilities for extending the linear dynamic range of the ESI process are also discussed.

\section{Experimental}

An Agilent MSD1100 single quadrupole mass spectrometer (Agilent Technologies, Palo Alto, CA) was used in these investigations. As shown in Figure 1, the standard ion inlet interface of the mass spectrometer was replaced with a heated capillary-ion funnel interface providing significantly improved sensitivity [22, 23]. The electrospray emitter was mounted on an $X-Y$ translation stage allowing fine adjustment of its position with respect to the heated capillary inlet. A syringe pump (KD Scientific, Holliston, MA) was used for sample infusion and a micro syringe $(100 \mu \mathrm{l})$ was connected to the ESI emitter through a metal union. A high voltage DC power supply connected to the LC union provided the DC potential necessary for ESI operation. ESI emitters were made by pulling sections of $50 \mu \mathrm{m}$ i.d. $/ 150 \mu \mathrm{m}$ o.d. fused silica capillary (Polymicro Technologies, Phoenix, AZ) down to approximately $15 \mu \mathrm{m}$ tips using a butane torch. The ion funnel used in this study was described in detail elsewhere [23, 24]. This ion funnel configuration was shown to have high efficiency in focusing and transmitting ions over a broad $m / z$ range $[22,23]$. Following the exit of the funnel is an electrostatic lens with a $1.93 \mathrm{~mm}$ orifice diameter which served as a conductance limiting orifice and provided RF shielding between the ion funnel and the octopole ion guide. The electrostatic lens was electrically insulated from the ion funnel electrode. The skimmer voltage of the MSD1100 was used to supply the DC 
voltage for the electrostatic lens allowing automatic optimization using the parameter ramping function of the MSD1100 instrument control software. To effectively disperse the gas expansion jet from the exit of the heated capillary inlet and significantly reduce the gas load down-stream of the ion funnel, a jet disrupter, consisting mainly of an electrically isolated metal disk of $6.5 \mathrm{~mm}$ in diameter, was installed on the center axis of the ion funnel $19.5 \mathrm{~mm}$ from the funnel entrance [23, 24]. The heated ESI inlet capillary assembly used a metal capillary (430 $\mu \mathrm{m}$ i.d. and $2.5 \mathrm{in}$. long) which was silver soldered into a metal block $[23,24]$. Two cartridge heaters were used to heat the capillary block and the temperature of the heated capillary was monitored by thermocouples and regulated using a close loop temperature control unit (Omega CN9000A). The temperature of the heated capillary inlet was fixed at $150^{\circ} \mathrm{C}$. A DC bias at $220 \mathrm{~V}$ was applied to the heated capillary block. The RF frequency and the amplitude applied to the ion funnel were $500 \mathrm{kHz}$ and $70 \mathrm{~V}_{p-p}$, respectively. The DC biases on the first ion funnel electrode and the last ion funnel electrode were approximately 200 and 35 $\mathrm{V}$, respectively, which resulted in an axial DC field of approximately $16 \mathrm{~V} / \mathrm{cm}$ in the ion funnel. The vacuum pressure was measured at 2.5 torr in ion funnel chamber, and $4.5 \times 10^{-6}$ torr in the MS analyzer chamber. The mass spectrometer was operated in the positive ESI mode. Selected ion monitoring mode of MS was used to measure the analyte ion intensity. During the experiment, both the electrospray voltage and the spray emitter position were carefully adjusted to achieve maximum MS sensitivity at each electrospray operating condition and a stereo zoom microscope was used to monitor electrospray stability. For the total electrospray current measurements at different flow rates, the mass spectrometer was replaced with a simple grounded metal plate connected to a picoammeter. The analytes used in this study included caffeine, MRFA (Met-Arg-Phe-Ala) and reserpine, which produce molecular ions at $\mathrm{m} / \mathrm{z} 195.1^{+}$for caffeine, $524.4^{+}$and $262.7^{++}$for MRFA, and $609.2^{+}$for reserpine. Samples having concentrations ranging from $10^{-7}$ to $10^{-3} \mathrm{M}$ for each analyte were used to study the MS responses at different electrospray flow rates. Mass spectra were acquired allowing the characterization of MS sensitivity and signal linearity at different sample concentrations. The three compounds were also mixed together at equimolar concentrations to study charge competition in the ESI process and its effects on the quantitative MS analysis. The selection of the analytes in this study is arbitrary but with the requirement that the compounds show different ionization efficiencies under the same experimental conditions, as discussed in detail below. However, the conclusions from this study are expected to be valid for any mixture.

\section{Results and Discussion}

Figure 2 shows MS signal intensity measurements for different electrospray infusion rates of reserpine solutions having concentrations ranging from $10^{-7} \mathrm{M}$ to

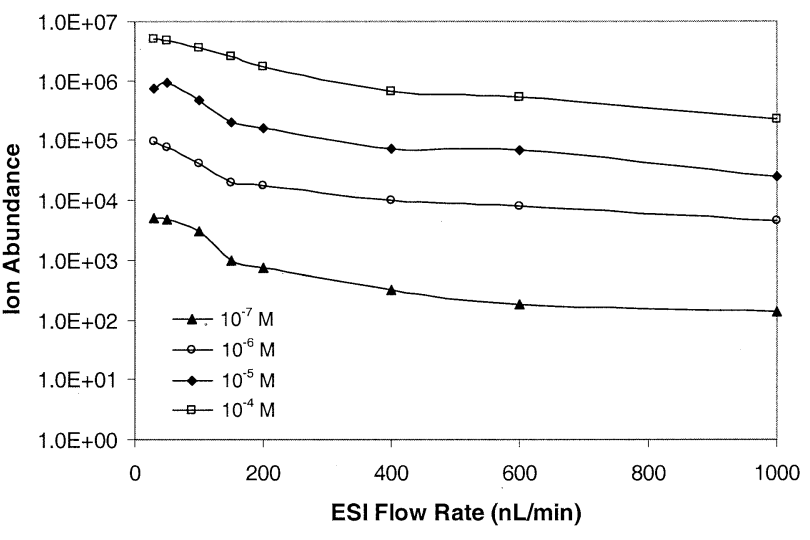

Figure 2. Ion abundance versus electrospray flow rate (in the semi log scale) for reserpine at different solution concentrations.

$10^{-4} \mathrm{M}$ in 50:50 methanol:water $+1 \%$ acetic acid. The MS operating parameters were fixed for each experiment and the electrospray voltage was adjusted at each flow rate to ensure stable cone-jet mode electrospray operation. As shown in Figure 2 and consistent with many previous reports, sensitivity increases as electrospray flow rate decreases for each solution concentration. The lower electrospray flow rate results in smaller charged droplets and ultimately provides a higher ionization efficiency. Over an order of magnitude sensitivity improvement was observed when the electrospray flow rate was reduced from $1 \mathrm{uL} / \mathrm{min}$ to $50 \mathrm{~nL} / \mathrm{min}$. The slight sensitivity decrease observed at the lowest flow rate $(30 \mathrm{~nL} / \mathrm{min})$ for the $10^{-5} \mathrm{M}$ concentration may be related to variations in ESI flow instability suggesting that an even finer emitter may be needed to maintain spray stability at the smallest flow rates where observation of electrospray becomes less effective for optimizing stability.

Figure 3 further compares the MS response for different electrospray flow rates using three sample solutions at the same concentration $10^{-5} \mathrm{M}$. Reserpine is shown to have significantly greater peak intensity at each flow rate compared to either caffeine or MRFA. Since all samples were prepared with the same solvent

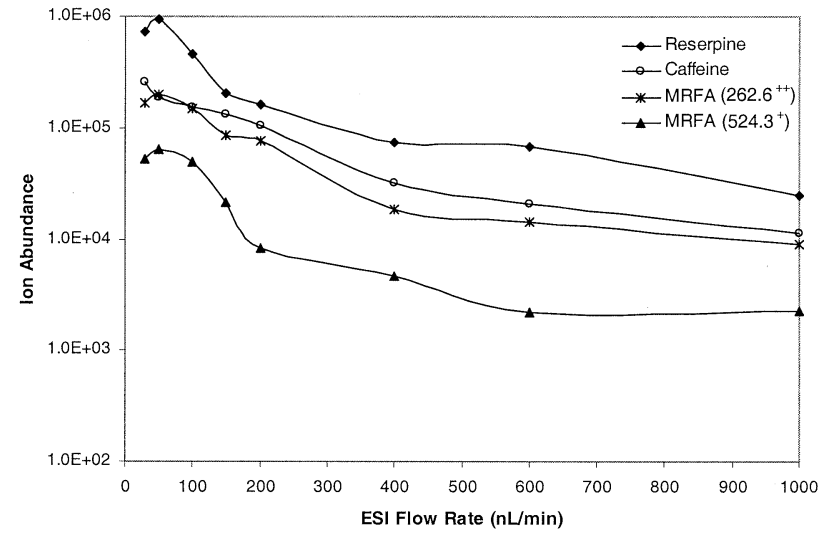

Figure 3. Signal intensities versus liquid flow rate (in the semi $\log$ scale) for pure $10^{-5} \mathrm{M}$ solutions of reserpine, caffeine, and MRFA. 


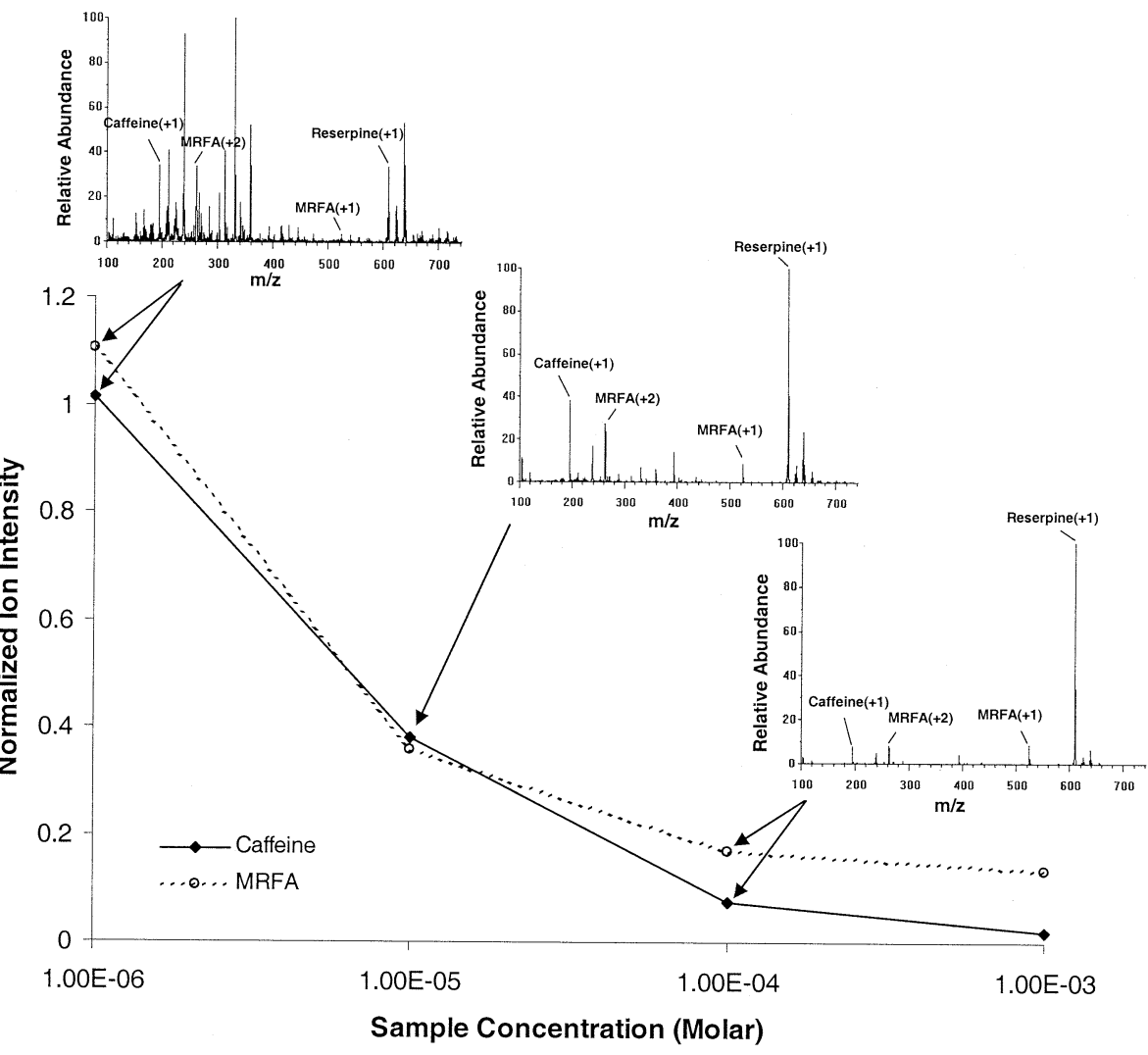

Figure 4. Normalized ion intensities of caffeine $\left(195.1^{+}\right)$and MRFA $\left(524.4^{+}, 262.7^{++}\right)$to the intensity of the reserpine $\left(609.2^{+}\right)$peak at different mixture concentrations. Representative mass spectra for the three lesser concentrations are also shown. Electrospray flow rate: $1 \mu \mathrm{l} / \mathrm{min}$.

mixture, the higher sensitivity implies a greater efficiency of the ESI process. This can either be attributed to charge competition of caffeine and MRFA with other solution components, or (more likely) greater reserpine surface activity. This result indicates that both caffeine and MRFA ESI-MS signals would likely be suppressed by reserpine in mixtures of the three compounds.

To investigate the charge competition, solutions having equimolar concentrations of reserpine, caffeine, and MRFA were prepared. Figure 4 shows the ion intensities of caffeine and MRFA (total intensity from both singly and doubly charged ions) relative to reserpine at different sample dilutions. At a concentration of $10^{-4}$ $\mathrm{M}$, the reserpine peak shows more than an order of magnitude greater intensity than caffeine or MRFA. The differences in peak intensity decrease as the solution concentration decreases to $10^{-5} \mathrm{M}$. As the sample become sufficiently dilute $\left(10^{-6} \mathrm{M}\right.$ for each analyte in the mixture), the relative ion intensities become nearly equivalent. Below this concentration we expect that MS response will be essentially linear with sample concentration for all the components in the mixture. This requires significantly improved MS sensitivity and better sample preparation with reduction of typical solvent contaminants to provide ESI-MS measurements in this regime.

To better understand these observations, an analysis was performed based upon the work of de la Mora and
Loscertales [8] who showed that for electrosprays of highly conductive liquids, the total electrospray current can be formulated as:

$$
I=\beta(\varepsilon)\left(\frac{Q k \gamma}{\varepsilon}\right)^{1 / 2}
$$

where $I$ is the total spray current or total excess charges in the electrospray, $k$ is the electric conductivity of the liquid, $\gamma$ is the surface tension of the liquid, $\varepsilon$ is the dielectric constant of the liquid, $\beta(\varepsilon)$ is an experimentally determined coefficient and $\mathrm{Q}$ is the liquid flow rate. Eq 1 was derived through a dimensional analysis of the charge transport process through the Taylor cone, and was experimentally verified by the authors using several mixtures.

For a solution containing $\mathrm{N}$ different compounds of interest with molar concentration $X_{N}$, if we assume that each compound becomes multiply charged in the electrospray ionization process, the total ion current corresponding to compound $\mathrm{J}$, when all the molecules of compound J are completely ionized, will be:

$$
I_{J}=e A_{V} Q \sum_{z=1}^{i(D)} z f_{z, J} X_{J}
$$


where $e$ is the electron charge $\left(1.6 \times 10^{-19}\right.$ Coulomb), $A_{V}$ is Avogadro's number, $f_{z, J}$ is the fraction of compound $J$ molecule ions carrying $z$ charges and $i(J)$ is the maximum charge carrying capacity of the compound $J$ ions. Both $f_{z, J}$ and $i(J)$ can be experimentally determined from the compound charge distribution in the mass spectra at given experimental conditions. Based on the definition of $f_{z, J}$, we also have:

$$
\sum_{z=1}^{i(D)} f_{z, J}=1
$$

The total ion current when all the analytes of interest in the solution are ionized is then:

$$
I_{A}=e A_{V} Q \sum_{J=1}^{N}\left(\sum_{z=1}^{i(D)} z f_{z, J} X_{J}\right)
$$

The $I_{A}$, defined by eq 4 represents an ideal condition in the electrospray ionization process. Although complete analyte ionization from solution may not be realized, the ratio between the total electrospray ion current $I$ and the analyte ion current $\mathrm{I}_{\mathrm{A}}$, as defined in eqs 1 and 4 , can still provide a measure of the probability for analyte ionization in ESI. Consequently, we define this ratio as the electrospray ionization charge capacity for a solution, which can be expressed as:

$$
C_{i}=\frac{I}{I_{A}}=\frac{\beta(\varepsilon)}{e A_{V} \sum_{J=1}^{N}\left(\sum_{z=1}^{i(J)} z f_{z, J} X_{J}\right)} \cdot\left(\frac{k \gamma}{\varepsilon Q}\right)^{\frac{1}{2}}
$$

Based upon eq 5 one can partition ESI-MS response into three limiting conditions defined here as: (1) $C_{i} \gg 1,(2)$ $C_{i} \sim 1$, and (3) $C_{i} \ll 1$. When $C_{i} \gg 1$, the sample solution is sufficiently dilute compared to the available excess electrospray charge. Here, ESI charge competition is expected to be negligible and the ESI-MS response is linear with concentration for each compound in the mixture. For $C_{i} \sim 1$, the total excess charge becomes comparable to the total number of analyte ions formed by ESI. ESI charge competition in this case is expected to be important. Compounds with different ionization efficiencies will have different MS responses. This can result in the different dynamic ranges for compounds in mixtures, as discussed below, and even the failure to detect some species in mixtures. Eventually, when sample concentration increases further $\left(C_{i} \ll 1\right)$ the MS response is expected to be substantially independent of sample concentration (i.e., saturated) [13].

To test this model, eqs $1-5$ were compared with the experimental data. Eq 1 indicates that the total electrospray current depends on both the liquid flow rate and the liquid properties. For both non-ionic and nonsurface active compounds, where solution surface ten-

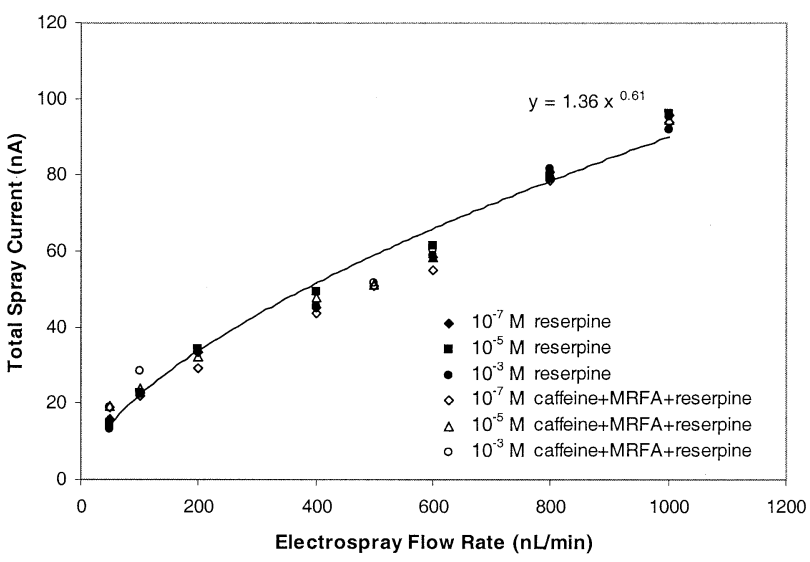

Figure 5. Total spray ion current versus electrospray flow rate for pure reserpine and mixtures of MRFA, reserpine and caffeine at different solution concentrations.

sion, electric conductivity and dielectric constant are not significantly altered, the total electrospray current is expected to depend only on the liquid flow rate. Figure 5 shows that the total electrospray currents remain essentially unchanged at a given flow rate for pure reserpine solutions, as well as a mixture of reserpine, caffeine and MRFA at different concentrations. Good agreement was also observed between the current measurements shown in Figure 5 and eq 1, implying that stable cone-jet mode electrosprays $[8,20]$ were effectively maintained in these experiments. Using the experimental data for the total electrospray current (Figure 5) both $I_{A}$ and $C_{i}$ can be calculated using eqs 4 and 5 for the conditions of Figure 4. The data given in Table 1 are also consistent with the results shown in Figure 4. At the concentration of $10^{-6} \mathrm{M}$, a very large $C_{i}$ value suggests negligible charge competition in the ESI process. The experiments confirm the comparable MS intensities for all compounds. As the sample concentration increases, $C_{i}$ decreases and charge competition becomes increasingly important. Compounds with higher ionization efficiencies show higher MS signal intensities. Comparing the results in Figure 4 and Table 1, we also conclude that charge competition in the ESI process will be generally negligible when $C_{i}$ is larger than $\sim 10$.

The $C_{i}$ in eq 5 can also be used to define the upper concentration limit for the ESI linear dynamic range. As

Table 1. Calculation of electrospray ionization capacity for the experimental results in Figure 4

\begin{tabular}{lccc}
\hline$X$ (Molar) $^{\mathrm{a}}$ & $\mathrm{I}(\mathrm{nA})^{\mathrm{b}}$ & $\mathrm{I}_{\mathrm{A}}(\mathrm{nA})^{\mathrm{c}}$ & $\mathrm{C}_{\mathrm{i}}$ \\
\hline \hline $10^{-4}$ & 95.1 & 560 & 0.17 \\
$10^{-5}$ & 95.1 & 60.6 & 1.57 \\
$10^{-6}$ & 95.1 & 6.28 & 15.2 \\
\hline
\end{tabular}

aThe molar concentrations listed represent the concentration for each compound in the mixture.

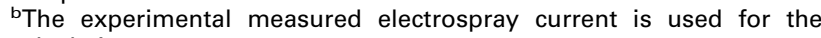
calculation.

${ }^{\mathrm{c} C}$ Calculated using eq 4. 


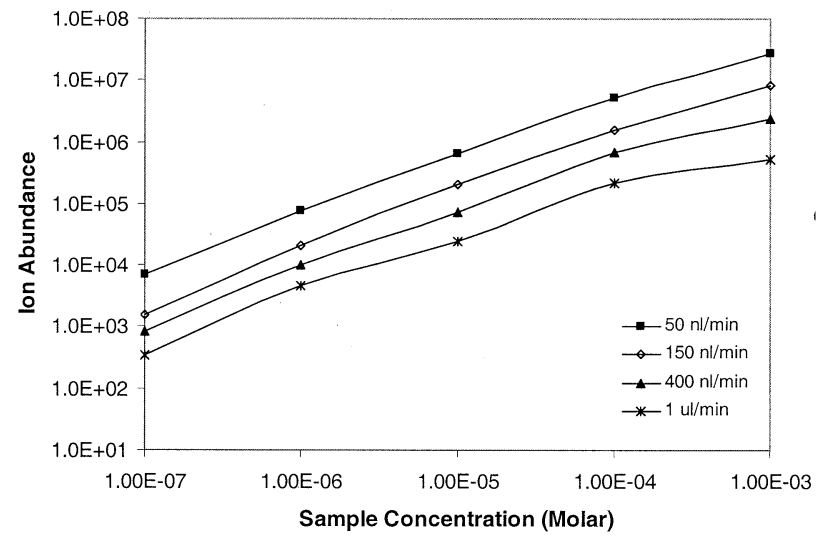

Figure 6. MS sensitivity versus sample concentration at different electrospray flow rates for a pure reserpine solution.

discussed above, the ESI-MS response is expected to become nonlinear, or even independent of the sample concentration, when $C_{i}$ becomes significantly small. It is interesting to note that $C_{i}$ in eq 5 is inversely proportional to the square root of the electrospray flow rate. While perhaps counter intuitive, this implies that $a$ smaller electrospray flow rate can provide a wider linear dynamic range. To test this prediction, the ESI dynamic range was measured experimentally using pure reserpine of different concentrations. Figure 6 shows the MS sensitivity as a function of reserpine concentration at different flow rates. Consistent with the prediction from eq 5, the linear dynamic range of ESI decreases as the electrospray flow rate increases. The calculation using the data in Figure 6 showed that the upper concentration limit for the ESI linear dynamic range corresponds approximately to $C_{i} \sim 1$.

The prediction for the ESI linear dynamic range was further tested using a mixture of caffeine, reserpine and MRFA having equimolar concentrations, since it was expected that charge competition in the ESI process could affect the linear dynamic range for each compound. Figure 7 shows the MS sensitivity for both

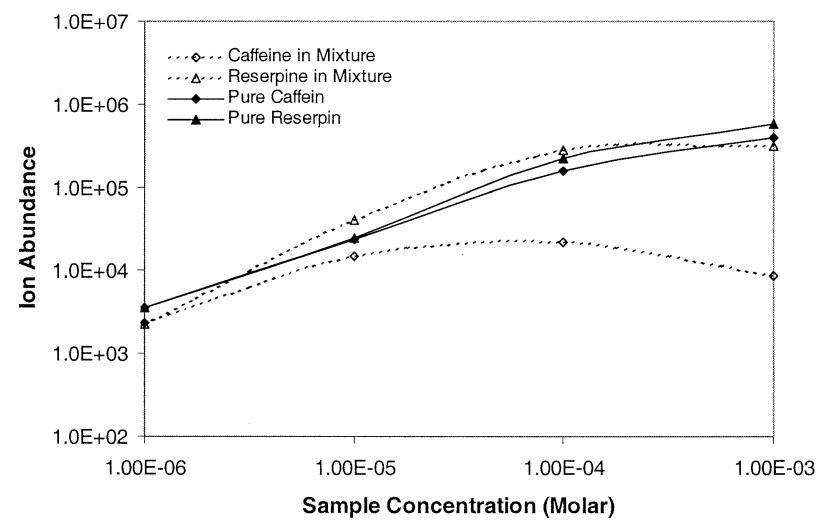

Figure 7. MS sensitivity versus sample concentration at a 1 $\mu \mathrm{L} /$ min electrospray flow rate for caffeine and reserpine in a mixture of MRFA, reserpine and caffeine (dashed lines), and reserpine, and caffeine as pure solutions (solid line). reserpine and caffeine at an electrospray flow rate of 1 $\mu \mathrm{L} / \mathrm{min}$. To compare the ESI dynamic range change as the sample complexity changes, the experimental data for reserpine and caffeine in the pure sample solution are also shown in Figure 7. The results indicate that the linear dynamic ranges for different compounds can be affected differently by the sample complexity. The dynamic range for reserpine, having the highest ionization efficiency for the three compounds, remained virtually the same for both the pure sample solution and the mixture. On the other hand, the dynamic range of caffeine in the mixture was significantly reduced.

A simple experimental approach for reducing or avoiding the nonlinear response for each analyte in the mixture is to dilute the samples. As shown in Table 1 and Figure 4, in cases where the total analyte concentration is approximately known, a $C_{i}$ value of $\sim 10$ can be conservatively used to predict the upper limit for ESI-MS linear dynamic range. A more effective way to achieve comparable ionization efficiencies for all the analytes in the mixture is to reduce electrospray flow rates. The benefits of operating in the nano-electrospray mode has been discussed extensively $[5,6,9,25,26]$.

The use of an ESI capacity $C_{i}$ value allows one to predict both the sample concentration limit for charge competition and the upper concentration limit for a linear dynamic range in ESI-MS. The model also suggests possible approaches for increasing the dynamic range in ESI-MS analyses (since $C_{i}$ is inversely proportional to the square root of the electrospray flow rate, the dynamic range can be increased by reducing flow rate). Operating in the nano-electrospray mode will not only increase the upper limit of the linear dynamic range, as indicated by eq 5 , but also decrease the lower concentration limit, which is essentially determined by the detection capabilities of the mass spectrometer.

We also expect that another attractive alternative for significantly extending ESI dynamic range is to operate an electrospray in a multi-spray mode. As shown in a previous investigation [27], when electrospray is operated in the multi-spray mode at a given flow rate $Q$, the total electrospray current is proportional to the square root of the number of the electrosprays. In this case,

$$
C_{i}=\frac{I_{\text {total }}}{I_{A}}=\frac{\sqrt{n} \beta(\varepsilon)}{e A_{V} \sum_{J=1}^{N}\left(\sum_{z=1}^{i(J)} z f_{z, J} X_{J}\right)} \cdot\left(\frac{k \gamma}{\varepsilon Q}\right)^{\frac{1}{2}}
$$

where $\mathrm{n}$ is the number of electrosprays at a given flow rate $\mathrm{Q}$. In eq 6, $C_{i}$ is proportional to the square root of the number of electrosprays. Since each electrospray in a multi-electrospray operates in a lower liquid flow rate, ESI dynamic range is extended due to the greater overall sensitivity at lower concentrations.

As a last example, the model established in this study can also be extended to capillary LC-ESI-MS application. The $C_{i}$ defined in eq 5 can be used to 
predict the ESI linear dynamic range where different LC columns are used for a given sample loading. The liquid flow rate in the LC column can be expressed as:

$$
Q=\frac{1}{4} \pi d_{c}^{2} V_{l}
$$

where $d_{c}$ is the column diameter, $V_{l}$ is the linear velocity of the liquid in the column. If we further assume the width of any chromatographic peak to be $l_{p}$ and consider a case where $\mathrm{N}$ different compounds co-elute (in one peak), the molar concentration during elution for compound $\mathrm{J}$ will then be:

$$
X_{J}=\frac{4 m_{J}}{\pi d_{c}^{2} l_{p} M_{J}}
$$

where $m_{\mathrm{J}}$ is the total mass for compound $\mathrm{J}$, which is related to the total sample mass loaded on LC column, $\mathrm{M}_{\mathrm{J}}$ is the molecular weight of the compound J. Substituting eqs 7 and 8 into eq 5 , we obtain:

$$
C_{i}=\frac{I}{I_{A}}=\frac{L_{p} \beta(\varepsilon)}{e A_{V} \sum_{J=1}^{N}\left(\sum_{z=1}^{i(N)} z f_{z, J} \frac{m_{J}}{M_{J}}\right)} \cdot\left(\frac{\pi k \gamma}{4 \varepsilon V_{1}}\right)^{\frac{1}{2}} d_{c}
$$

Although it is difficult to determine $\mathrm{m}_{\mathrm{J}}$, one can see from eq 9 that $C_{i}$ is proportional to the column diameter. This shows that the upper concentration limit of the LC-ESI-MS dynamic range can be extended by using a larger diameter LC column at a given sample mass loading and the same separation efficiency (i.e., constant $\mathrm{V}_{\mathrm{l}}$ and $\mathrm{L}_{\mathrm{p}}$ ). However, the apparent benefit disappears when sample size is scaled with flow rate, and it should be noted that a smaller diameter column will always provide higher ionization efficiency and be attractive for cases where sample size is limited [28, 29].

\section{Conclusions}

Large increases in ESI-MS sensitivity occur as electrospray flow rate decreases. The importance of charge competition in the ESI process for a given operating condition is determined by the relative magnitude of available excess charge in the electrospray and the total number of analyte ions in the solution. When a sufficiently diluted sample is used, the charge competition becomes negligible and MS sensitivities similar.

The ESI dynamic range shows different characteristics depending on the complexity of the sample solutions. For a pure sample solution, the maximum concentration limit is determined by the total excess charge available in electrospray to ionize the analyte molecules, which is consistent with the conclusion reached by others [11-13]. However, in the case of sample mixtures, the ESI dynamic range can be affected by charge competition. A theoretical analysis introducing an ESI charge capacity appears to predict both the onset of significant charge competition and the upper concentration for linear ESI-MS dynamic range. The present analysis also shows that ESI dynamic range can be potentially extended using multi-electrospray sources.

The present work highlights several advantages to use very low flow rate separations with ESI-MS. First, smaller electrospray flow rates can provide a wider linear dynamic range. It is expected that lower flow rate nano-electrosprays will approach a regime where ionization efficiencies will become similar for any compound, where analytes compete effectively with solvent related species for charge, and ionization efficiencies will approach $100 \%$. While operation under such conditions poses challenges for both the separations and MS sensitivity, recent results have demonstrated the feasibility of such approaches for ultra-sensitive and broad dynamic range studies of complex proteome samples $[28,29]$.

\section{Acknowledgments}

The authors thank the National Institutes of Health National Center for Research Resources (RR18522) for support of this work. The Pacific Northwest National Laboratory is operated by Battelle Memorial Institute for the U.S. Department of Energy through contract DE-AC06-76RLO 1830.

\section{References}

1. Yamashita, M.; Fenn, J. B. Electrospray Ion Source. Another Variation on the Free-Jet Theme. J. Phys. Chem. 1984, 88, 4451-4459.

2. Fenn, J. B.; Mann, M.; Meng, C. K.; Wong, S. F.; Whitehouse, C. M. Electrospray Ionization-Principles and Practice. Mass Spectrom. Rev. 1990, 9, 37-70.

3. Smith, R. D.; Loo, J. A.; Loo, R. R. O.; Busman, M.; Udseth, H. R. Principles and Practice of Electrospray Ionization MassSpectrometry for Large Polypeptides and Proteins. Mass Spectrom. Rev. 1991, 10, 359-451.

4. Cech, N. B.; Enke, C. G. Practical Implications of Some Recent Studies in Electrospray Ionization Fundamentals. Mass Spectrom. Rev. 2001, 20, 362-387.

5. Wilm, M.; Mann, M. Analytical Properties of the Nanoelectrospray Ion Source. Anal. Chem. 1996, 68, 1-8.

6. Schmit, A.; Karas, M.; Dulcks, T. Effect of Different Solution Flow Rates on Analyte Ion Signals in Nano-ESI MS, or: When Does ESI Turn Into Nano-ESI? J. Am. Soc. Mass Spectrom. 2003, 14, 492-500.

7. Wilm, M.; Mann, M. Electrospray and Taylor-Cone Theory, Dole's Beam of Macromolecules at Last? Int. J. Mass Spectrom. Ion Processes 1994, 136, 167-180.

8. Fernandez de la Mora, J.; Loscertales, I. G. The Current Emitted by Highly Conducting Taylor Cones. J. Fluid Mech. 1994, 260, 155-184.

9. Juraschek, R.; Dulcks, T.; Karas, M. Nanoelectrospray-More Than Just a Minimized-Flow Electrospray Ionization Source. J. Am. Soc. Mass Spectrom. 1999, 10, 300-308.

10. Gabelica, V.; Vreuls, C.; Filee, P.; Duval, V.; Joris, B.; De Pauw, E. Advantages and Drawbacks of Nanospray for Studying Noncovalent Protein-DNA Complexes by Mass Spectrometry. Rapid Commun. Mass Spectrom. 2002, 16, 1723-1728. 
11. Tang, L.; Kebarle, P. Dependence of Ion Intensity in Electrospray Mass Spectrometry on the Concentration of the Analytes in the Electrosprayed Solution. Anal. Chem. 1993, 65, 3654-3668.

12. Kebarle, P.; Tang, L. From Ions in Solution to Ions in the Gas-Phase-The Mechanism of Electrospray Mass-Spectrometry. Anal. Chem. 1993, 65, 972A-986A.

13. Enke, C. G. A Predictive Model for Matrix and Analyte Effects in Electrospray Ionization of Singly-Charged Ionic Analytes. Anal. Chem. 1997, 69, 4885-4893.

14. Kostiainen, R.; Bruins, A. P. Effect of Solvent on Dynamic Range and Sensitivity in Pneumatically-Assisted Electrospray (Ion Spray) Mass Spectrometry. Rapid Commun. Mass Spectrom. 1996, 10, 1393-1399.

15. Zook, D. R.; Bruins, A. P. On Cluster Ions, Ion Transmission, and Linear Dynamic Range Limitations in Electrospray (Ionspray) Mass Spectrometry. Int. J. Mass Spectrom. Ion Processes $1997,162,129-147$.

16. Xu, X.; Nolan, S. P.; Cole, R. B. Electrochemical Oxidation and Nucleophilic-Addition Reactions of Metallocenes in Electrospray Mass-Spectrometry. Anal. Chem. 1994, 66, 119-125.

17. Cech, N. B.; Enke, C. G. Relating Electrospray Ionization Response to Nonpolar Character of Small Peptides. Anal. Chem. 2000, 72, 2717-2723.

18. Cloupeau, M.; Prunet-Foch, B. Electrostatic Spraying of Liquids in Cone-Jet Mode. J. Electr. 1989, 22, 135-159.

19. Chen, D. R.; Pui, D. Y. H.; Kaufman, S. L. Electrospraying of Conducting Liquids for Monodisperse Aerosol Generation in the $4 \mathrm{nM}$ to $1.8 \mathrm{mU}-\mathrm{M}$ Diameter Range. J. Aerosol Sci. 1995, 26, 963-977.

20. Hartman, R. P. A.; Brunner, D. J.; Camelot, M. A.; Marijnissen, J. C. M.; Scarlett, B. Electrohydrodynamic Atomization in the Cone-Jet Mode Physical Modeling of the Liquid Cone and Jet. J. Aerosol Sci. 1999, 30, 823-849.

21. Tang, K.; Smith, R. D. Physical/Chemical Separations in the Breakup of Highly Charged Droplets from Electrosprays. J. Am. Soc. Mass Spectrom. 2001, 12, 343-347.
22. Tolmachev, A. V.; Kim, T.; Udseth, H. R.; Smith, R. D.; Bailey, T. H.; Futrell, J. H. Simulation-Based Optimization of the Electrodynamic Ion Funnel for High Sensitivity Electrospray Ionization Mass Spectrometry. Int. J. Mass Spectrom. 2001, 203, 31-47.

23. Kim, T.; Tang, K.; Udseth, H. R.; Smith, R. D. A Multicapillary Inlet Jet Disruption Electrodynamic Ion Funnel Interface for Improved Sensitivity Using Atmospheric Pressure Ion Sources. Anal. Chem. 2001, 73, 4162-4170.

24. Tang, K.; Tolmachev, A. V.; Nikolaev, E.; Zhang, R.; Belov, M. E.; Udseth, H. R.; Smith, R. D. Independent Control of Ion Transmission in a Jet Disrupter Dual-Channel Ion Funnel Electrospray Ionization MS Interface. Anal. Chem. 2002, 74, 5431-5437.

25. Smith, R. D.; Shen, Y.; Tang, K. Ultrasensitive and Quantitative Analyses from Combined Separations-Mass Spectrometry for the Characterization of Proteomes. Acc. Chem. Res. 2004, 37, 269-278.

26. Znamenskiy, V.; Marginean, I.; Vertes, A. Solvated Ion Evaporation from Charged Water Nanodroplets. J. Phy. Chem. 2003, 107, 7406-7412.

27. Tang, K.; Lin, Y.; Matson, D. W.; Kim, T.; Smith, R. D. Generation of Multiple Electrosprays Using Microfabricated Emitter Arrays for Improved Mass Spectrometric Sensitivity. Anal. Chem. 2001, 73, 1658-1663.

28. Shen, Y.; Moore, R. J.; Zhao, R.; Blonder, J.; Auberry, D. L.; Masselon, C.; Pasa-Tolic, L.; Hixson, K. K.; Auberry, K. J.; Smith, R. D. High-Efficiency On-Line Solid-Phase Extraction Coupling to 15-150-Microm-i.d. Column Liquid Chromatography for Proteomic Analysis. Anal. Chem. 2003, 75, 3264-3273.

29. Shen, Y.; Tolic, N.; Masselon, C.; Pasa-Tolic, L.; Camp, D. G. II.; Hixson, K. K.; Zhao, R.; Anderson, G. A.; Smith, R. D. Ultrasensitive Proteomics Using High-Efficiency On-Line Micro-SPE-NanoLC-NanoESI MS and MS/MS. Anal. Chem. 2004, $76,144-154$ 